\title{
Mid-infrared electric field sampling approaching single-photon sensitivity
}

\author{
C. Hofer ${ }^{1,2}$, D. Gerz ${ }^{1,2}$, M. Högner ${ }^{1}$, T. P. Butler ${ }^{3}$, C. Gaida ${ }^{4}$, T. Heuermann ${ }^{5,6}$, M. Gebhardt ${ }^{5,6}$, \\ N. Karpowicz ${ }^{8}$, J. Limpert ${ }^{5,6,7}$, F. Krausz ${ }^{1,2}$, I. Pupeza ${ }^{1,2}$ \\ 1. Max Planck Institute of Quantum Optics, Hans-Kopfermann-Str. 1, D-85748 Garching, Germany \\ 2. Ludwig Maximilians University Munich, Faculty of Physics, Am Coulombwall 1, D-85748 Garching, Germany \\ 3. University of Maryland, Institute for Research in Electronics and Applied Physics, College Park, MD, United States \\ 4. Active Fiber Systems GmbH, Ernst-Ruska Ring 11, D-07745 Jena, Germany
}

5. Institute of Applied Physics, Abbe Centre of Photonics, Friedrich-Schiller Univ. Jena, Albert-Einstein Str. 15, D-07745 Jena, Germany

6. Helmholtz-Institute Jena, Frobelstieg 3, D-07743 Jena, Germany

7. CNR NANOTEC Institute of Nanotechnology, IT-73100 Lecce, Italy

8. Fraunhofer Institute for Applied Optics and Precision Engineering, Albert-Einstein Str. 7, D-07745 Jena, Germany

Electro-optic sampling (EOS) [1,2] enables measurements of light fields with sub-cycle temporal resolution. Here, we show the feasibility of mid-infrared (MIR), field-resolved detection with nearly single-photon sensitivity. This is facilitated by the thorough optimization of our high-power, Thulium (Tm)-based laser system and the EOS detection [3]. The experimental setup is shown in Fig. 1a and is described in detail in Ref. [4].
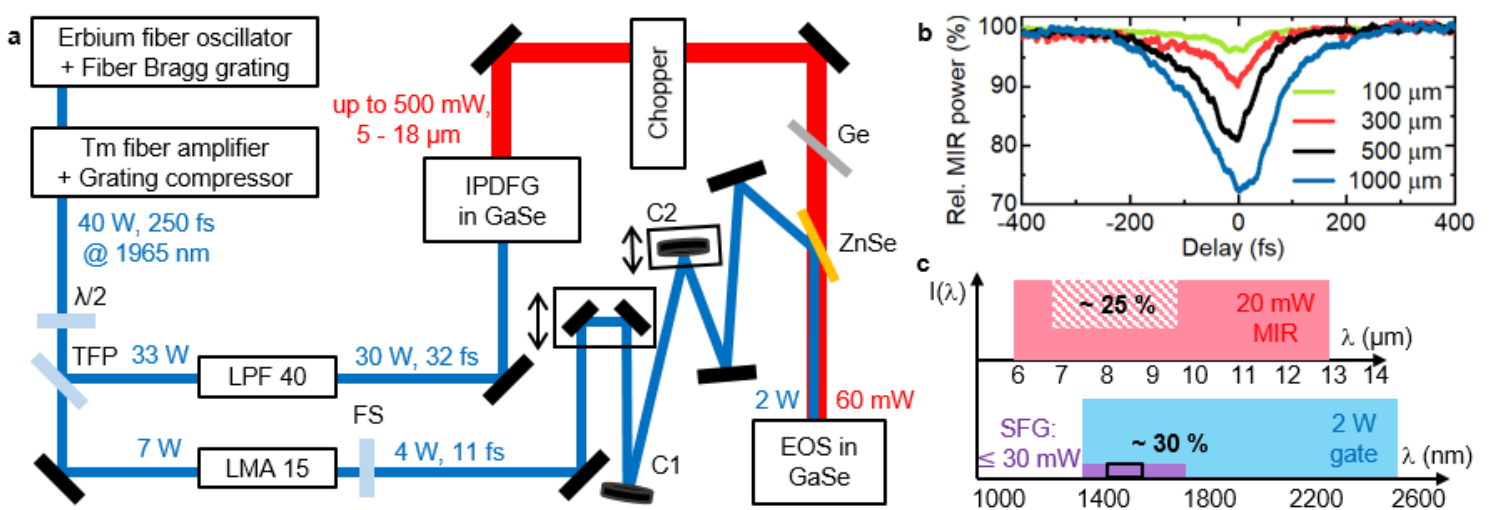

Fig. 1 (a) Experimental setup. TFP: Thin film polarizer, LPF: Large pitch fiber, LMA: Large mode area, C1, C2: concave mirrors. (b) Results of MIR depletion measurements for several EOS crystal thicknesses. (c) Sketch of the overall MIR photon detection efficiency.

The output from the Tm-fiber amplifier is further compressed with photonic crystal fibers in two channels. $30 \mathrm{~W}$, 32-fs-pulses are used to drive intra-pulse difference frequency generation (IPDFG) in Gallium Selenide (GaSe), generating high-power, few-cycle MIR transients. A mechanical chopper modulates the beam at $9 \mathrm{kHz}$ to allow for lock-in detection of the EOS traces. We scan the delay of the $2 \mathrm{~W}, 11 \mathrm{fs}$ EOS gate pulses with a mechanical stage. A telescope, consisting of two concave mirrors, is used to optimize the spatial overlap of the MIR fields and gate pulses in the EOS crystal. They are collinearly combined for EOS detection with an uncoated Zinc Selenide (ZnSe) plate in Brewster's angle.

We characterize the detection efficiency by measuring the delay-dependent MIR depletion (Fig. 1b). The maximum, spectrally integrated up-conversion efficiency is $25 \%$, for a $1 \mathrm{~mm}$ thick GaSe EOS crystal. A spectral filter is employed for optimization of the detection dynamic range [5], allowing - together with reflection losses for the detection of $\sim 30 \%$ of all sum-frequency photons. As indicated in Fig. 1c, the spectrally-resolved depletion surpasses $50 \%$. Therefore, for those wavelengths with largest depletion, and with balanced detection close to the near-infrared shot noise limit, MIR photon numbers as low as 10/second can be detected in a field-resolved manner.

Together with record MIR-powers ( $>10^{18}$ photons/second at the detector), this paves the way to linear, fieldresolved measurements over up to 9 (field) and 18 (intensity) orders of magnitude. This unprecedented sensitivity and dynamic range allow us to push field-resolved spectroscopy [6] in the MIR to its fundamental limits.

\section{References}

[1] Q. Wu and X.-C. Zhang, "Free-space electro-optic sampling of terahertz beams," Appl. Phys. Lett. 67, 3523 (1995).

[2] G. Gallot and D. Grischkowsky, "Electro-optic detection of terahertz radiation,” J. Opt. Soc. Am. B 16, 1204 (1999).

[3] C. Hofer et al., "Quantum-Efficiency and Bandwidth Optimized Electro-Optic Sampling," CLEO Europe (2019), DOI: 10.1109/CLEOEEQEC.2019.8873083.

[4] T. P. Butler et al., "Watt-scale 50-MHz source of single-cycle waveform-stable pulses in the molecular fingerprint region," Opt. Lett. 44, 1730 (2019).

[5] M. Porer et al., "Shot noise reduced terahertz detection via spectrally postfiltered electro-optic sampling," Opt. Lett. 39, 2435 (2014).

[6] I. Pupeza et al., "Field-resolved infrared spectroscopy of biological systems," Nature 577, 52 (2020). 